\title{
Orbit of a lunar artificial satellite: Analytical theory of perturbations
}

\author{
B. De Saedeleer and J. Henrard \\ Department of Mathematics, FUNDP, Namur, B-5000, Belgium \\ email: bernard.desaedeleer@fundp.ac.be, jacques.henrard@fundp.ac.be
}

\begin{abstract}
We are currently developing an analytical theory of an artificial satellite of the Moon. It is an interesting problem because the dynamics of a lunar orbiter is quite different from that of an artificial satellite of the Earth, by at least two aspects: the $J_{2}$ lunar gravity term is only $1 / 10$ of the $C_{22}$ term and the third body effect of the Earth on the lunar satellite is much larger than the effect of the Moon on a terrestrial satellite. So we have to account at least for these larger perturbations. We use here the method of the Lie Transform as perturbation method. The Hamiltonian of the problem is first averaged over the fast angle, in canonical variables. The solution is developed in powers of the small factors linked to $n_{\mathbb{1}}, J_{2}, C_{22}$ and to the Earth's position. The Earth location is determined by the lunar theory ELP2000 (Chapront-Touzé \& Chapront 1991) from which we take the leading terms. Series developments are made with our home-made Algebraic Manipulator, the MM (standing for "Moon's series Manipulator"). The results are obtained in a closed form, without any series developments in eccentricity or inclination. So the solution applies for a wide range of values, except for few isolated critical values. We Achieved, among others, second order results for the combined effect of $J_{2}$ and $C_{22}$. As a side result, we were able to check the second order generator $\mathcal{W}_{2}$ given by Kozai for the effect of the $J_{2}$ term on an artificial satellite.
\end{abstract}

\section{Introduction}

The case study of a satellite around the Moon is quite different from the one around the Earth on several aspects. First of all, the moon is a slowly rotating body and has no dense atmosphere. Secondly, as it is well known, the lunar gravity field is far from being central, nor does it exhibit any strong symmetry of revolution; see e.g. Konopliv (2001) for a recent model in spherical harmonics. The order of magnitude of the second order coefficients for the Earth (Kaula 1966) and the Moon (Bills \& Ferrari 1980) is given in the Table 1. The Moon is much less flattened than the Earth, which makes the $C_{22}$ coefficient to come closer to $J_{2}$ (at 1 order of magnitude instead of 3 in the case of the Earth); so it needs to be considered. Moreover, the effect of the Earth on the lunar satellite is much larger than the effect of the Moon on a terrestrial satellite; so the former effect is mixed to the effects of the shape of the lunar gravity field. In this paper, we will focus on the combined effect of the perturbations $J_{2}$ and $C_{22}$, and introduce the effect of the Earth considered as a third body.

Table 1. Some orders of magnitude for $J_{2}$ and $C_{22}$

\begin{tabular}{ccc}
\hline & $C_{20} \equiv-J_{2}$ & $C_{22}$ \\
\hline Earth $\oplus$ & $-10^{-3}$ & $2.10^{-6}$ \\
Moon $₫$ & $-2.10^{-4}$ & $2.10^{-5}$ \\
\hline
\end{tabular}


The following assumptions have been made: the orbit of the Moon is circular (we neglect $e_{\mathbb{8}} \approx 0.055$ ); the motion of the Moon is uniform (librations are neglected); the lunar equator lies in the ecliptic (we neglect the inclination of the lunar equator to the ecliptic of $1.5^{\circ}$, and the inclination of the lunar orbit to the ecliptic of about $5^{\circ}$ ); the perturbation of the Sun is negligible; and the longitude of the lunar longest meridian $\lambda_{22}$ is equal to the longitude of the Earth $\lambda_{\oplus}$ (librations are neglected). This last assumption is one of the well known Cassini's laws (Cook 1988), stating that the Moon is in synchronous rotation: she rotates about her axis perpendicular to the plane of her orbit at an angular velocity $\gamma_{\S}$ that is equal to her mean angular velocity in her orbit $n_{\S}$; that is to say $\gamma_{\mathbb{Q}}=n_{\mathbb{8}}=2 \pi / T_{\mathbb{}}$ with the sidereal rotation period of the Moon being $T_{\mathbb{}}=27.3216615$ solar days.

\section{Partial perturbative Hamiltonians}

We work within the frame of the Hamiltonian formalism and use the classical Delaunay canonical variables $\left(q_{i}, p_{i}\right)=(l, g, h, L, G, H)$. The term $\frac{1}{2} v^{2}-\frac{\mu}{r}$ is then simply written $\mathcal{H}_{0}^{(0)}=-\frac{\mu^{2}}{2 L^{2}}$ (the unperturbed potential); next one has to develop the perturbations.

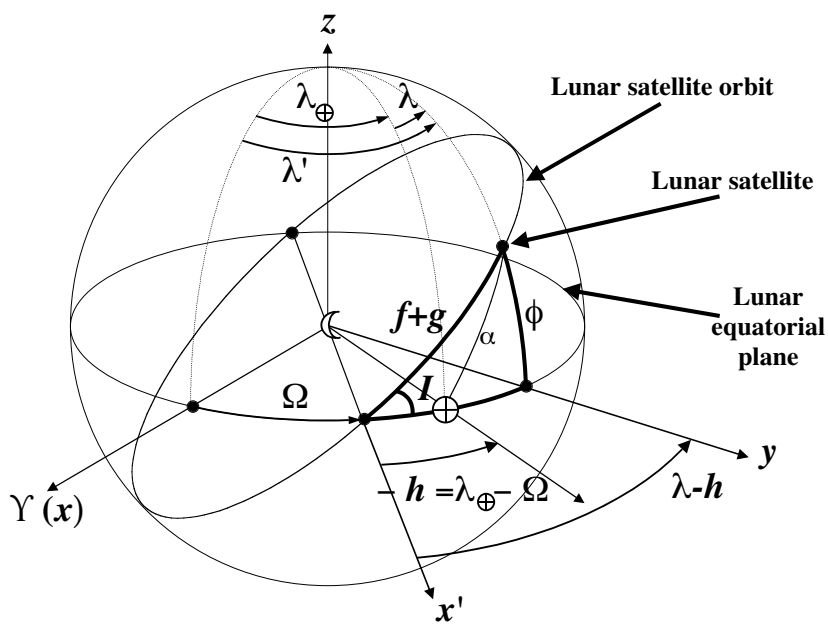

Figure 1. Simplified selenocentric sphere.

We define an inertial frame $(x, y, z)$ as follows (see Fig. 1): the origin is taken at the center of the Moon; the $x$ direction is the one of the first point of Aries $\Upsilon$, the $y$ is the direction normal to $x$ and contained in the lunar equatorial plane containing $x$, and the $z$ direction is the right-handed normal to $(x, y)$. We define also the spherical coordinates $\left(r, \lambda^{\prime}, \phi\right)$. The zonal perturbation in $J_{2}$ is defined as usual by $\epsilon \mathcal{H}_{2}^{(0)}=\epsilon \frac{\mu}{r^{3}} P_{20}(\sin \phi)$ where we use $\epsilon=J_{2} R^{2}$ and the Legendre Associated Functions $P_{n m}$. The argument $(\sin \phi)$ may partially be translated into Delaunay variables by way of spherical trigonometry (see Fig. 1, where the plane of the orbit is at an inclination $I): \sin \phi=\sin I \sin (f+g)$.

The sectorial perturbation in $C_{22}$ requires us to define $\lambda_{22}$ as the longitude of the lunar longest meridian (minimum inertia), which is $\lambda_{22}=\lambda_{\oplus}$. Since this angle rotates at the rate of the synchronous rotation $\dot{\lambda}_{\oplus}=n_{\mathbb{\bigotimes}}$, we preferably introduce a rotating frame by defining $\lambda=\lambda^{\prime}-\lambda_{\oplus}$ and also $h=\Omega-\lambda_{\oplus}$. A new term must then be added to the Hamiltonian in order to have $\dot{h}=\partial \mathcal{H} / \partial H=-n_{\S}$; we call this term $\mathcal{H}_{1}^{(0)}=-n_{\S} H$. Now the sectorial perturbation may be written as $\delta \mathcal{H B}_{2}^{(0)}=\delta \mu r^{-3} P_{22}(\sin \phi) \cos (2 \lambda)$, where 
we define $\delta=-C_{22} R^{2}$. We then use again some spherical trigonometry to switch into the canonical variables and we introduce the useful shortcuts $(s, c)=(\sin I, \cos I)$.

And last, we express the perturbation of the Earth as usual for a third body by $\gamma \mathcal{H} \mathcal{E}_{2}^{(0)}=\gamma a_{\oplus}^{3} r_{\oplus}^{-3} r^{2} P_{20}(\cos \psi), \psi$ being the angle between the Earth and the satellite, and with $\gamma=-\mu_{\oplus} a_{\oplus}^{-3}$. In summary, we will write in our case:

$$
\mathcal{H}^{(0)}=\mathcal{H}_{0}^{(0)}+\mathcal{H}_{1}^{(0)}+\epsilon \mathcal{H}_{2}^{(0)}+\delta \mathcal{H B}_{2}^{(0)}+\gamma \mathcal{H} \mathcal{E}_{2}^{(0)}
$$

along with the definitions:

$$
\begin{aligned}
\mathcal{H}_{1}^{(0)}= & -n_{\triangleleft} H \\
\mathcal{H}_{2}^{(0)}= & \frac{\mu}{4 r^{3}}\left(1-3 c^{2}-3 s^{2} \cos (2 f+2 g)\right) \\
\mathcal{H B}_{2}^{(0)}= & \frac{3 \mu}{4 r^{3}}\left\{2 s^{2} \cos (2 h)+(c+1)^{2} \cos (2 f+2 g+2 h)\right. \\
& \left.+(c-1)^{2} \cos (2 f+2 g-2 h)\right\} \\
\mathcal{H E}_{2}^{(0)}= & a_{\oplus}^{3} r_{\oplus}^{-3} r^{2} P_{20}(\cos \psi)
\end{aligned}
$$

Note that we directly choose to class the perturbations by their order of magnitude: $n_{\S}$ is first order, while the others are second order.

We come back to the choice of the variables now. There remains the variable $r$ and $f$ to be expressed as a function of $(l, g, h)$ in order to be able to apply a canonical perturbation method. It turns out that the functions $r=r(l, g, h)$ and $f=f(l, g, h)$ cannot be expressed in a closed form; so we prefer to use the following set of auxiliary variables $(\xi, f, g, h, a, n, e, \eta, s, c)$, which is closed and allow high eccentricities:

$$
\begin{array}{rlrl}
\xi & =\frac{a}{r}=\frac{1+e \cos f}{1-e^{2}}=\frac{1}{1-e \cos E} & & f \\
a & =\frac{L^{2}}{\mu} & n & =\frac{\mu^{2}}{L^{3}} \\
e & =\sqrt{1-\left(\frac{G}{L}\right)^{2}} & & =\sqrt{1-e^{2}}=\frac{G}{L} \\
s & =\sin I=\sqrt{1-\left(\frac{H}{G}\right)^{2}} & c & c \cos I=\frac{H}{G} \\
g & & h
\end{array}
$$

The only drawback of this set (2.6) is that it is redundant and that we need to perform partial derivatives of them with respect to the canonical variables $(l, g, h, L, G, H)$; but it is not too burdensome; the result is given in the Table 2. We have for example:

$$
\begin{aligned}
& \frac{d A}{d l}=\frac{\partial A}{\partial l}+\frac{\partial A}{\partial \xi} \quad \frac{\partial \xi}{\partial l}+\frac{\partial A}{\partial f} \quad \frac{\partial f}{\partial l} \\
& =\frac{\partial A}{\partial l}+\frac{\partial A}{\partial \xi}\left(\frac{-\xi^{2} e \sin f}{\eta}\right)+\frac{\partial A}{\partial f}\left(\xi^{2} \eta\right)
\end{aligned}
$$

Note that the quantity $\frac{\partial f}{\partial l}=\xi^{2} \eta$ plays an important role, since it will allow us to switch the integration from $l$ to $f$. In this new set of variables (2.6), the factor $\mu r^{-3}$ appearing in (2.3) and(2.4) may be written $\xi^{3} n^{2}$.

\section{Development of the third body perturbation}

We come back to the expression (2.5) of the perturbation of the Earth $\mathcal{H E}$. The $\cos \psi$ may be computed with: 
Table 2. Table of partial derivatives

\begin{tabular}{|c|c|c|c|c|}
\hline & $\partial / \partial L$ & $\partial / \partial G$ & $\partial / \partial H$ & $\partial / \partial l$ \\
\hline$\xi$ & $\frac{\xi^{2} \eta^{2}}{n a^{2} e} \cos f$ & $-\frac{\xi^{2} \eta}{n a^{2} e} \cos f$ & 0 & $-\frac{\xi^{2} e}{\eta} \sin f$ \\
\hline$a$ & $\frac{2}{a n}$ & 0 & 0 & 0 \\
\hline$n$ & $-\frac{3}{a^{2}}$ & 0 & 0 & 0 \\
\hline$s$ & 0 & $\frac{c^{2}}{n a^{2} \eta s}$ & $-\frac{c}{n a^{2} \eta s}$ & 0 \\
\hline$c$ & 0 & $-\frac{c}{n a^{2} \eta}$ & $\frac{1}{n a^{2} \eta}$ & 0 \\
\hline$e$ & $\frac{\eta^{2}}{n a^{2} e}$ & $-\frac{\eta}{n a^{2} e}$ & 0 & 0 \\
\hline$\eta$ & $-\frac{\eta}{n a^{2}}$ & $\frac{1}{n a^{2}}$ & 0 & 0 \\
\hline$f$ & $\frac{1+\xi \eta^{2}}{n a^{2} e} \sin f$ & $-\frac{1+\xi \eta^{2}}{\eta n a^{2} e} \sin f$ & 0 & $\xi^{2} \eta$ \\
\hline
\end{tabular}

$$
\begin{aligned}
\cos \psi=\frac{\vec{r} \cdot \vec{r}_{\oplus}}{r r_{\oplus}}= & A_{\oplus}(\cos h \cos (f+g)-c \sin h \sin (f+g)) \\
& +B_{\oplus}(\sin h \cos (f+g)+c \cos h \sin (f+g))+C_{\oplus} s \sin (f+g)
\end{aligned}
$$

where $\vec{A}_{\oplus}=\left(A_{\oplus}, B_{\oplus}, C_{\oplus}\right)$ is the direction of the Earth from the Moon. We now use the lunar theory ELP2000 (Chapront-Touzé \& Chapront 1991), which gives the position of the Moon with respect to the Earth, in the spherical coordinates $(V, U, R)$, where $V$ is the geocentric longitude ( $U$ and $V$ are referred to the mean dynamical ecliptic and mean equinox of date).

The position of the Moon is described by a series of periodic functions mainly of the fundamental arguments $D, l^{\prime}, l, F$, but also of the arguments of the other planets $M e, V e, T e, M a, J u, S a$. All these arguments are taken as linear function of time. We recall that $D$ is the secular part (nonperiodic part) of the difference between the mean longitude of the Moon and the geocentric mean longitude of the Sun, $l^{\prime}$ is the secular part of the geocentric mean anomaly of the Sun, $l$ is the secular part of the mean anomaly of the Moon, and $F$ is the secular part of the difference between the mean longitude of the Moon and the longitude of its ascending node on the mean ecliptic of date.

The authors of ELP2000 give the number of terms to take into account for each series in order to achieve several levels of precision; we follow the low precision recommendations, since we do not need a very long term accuracy: the duration of a lunar mission is typically much less than a century. So we will take only some leading terms and use the following simplified expressions for $U$ and $V$ :

$$
V=L+\alpha \quad \text { and } \quad U=\beta \quad \text { with } \quad \alpha=S_{V}+10^{-3} S_{V}^{\prime} \quad \text { and } \quad \beta=S_{U}
$$


and with

$$
\begin{aligned}
S_{V} & =\sum_{n=1}^{N_{V}=29} v_{n} \sin \left(i_{1, n} l+i_{2, n} D+i_{3, n} l^{\prime}+i_{4, n} F\right) \\
S_{V}^{\prime} & =v_{1}^{\prime} \sin \left(18 V e-16 T e-l+26.5426^{\circ}\right) \\
S_{U} & =\sum_{n=1}^{N_{U}=14} v_{n} \sin \left(j_{1, n} l+j_{2, n} D+j_{3, n} l^{\prime}+j_{4, n} F\right)
\end{aligned}
$$

from which we can then deduce

$$
\begin{aligned}
& A_{\oplus}=-\cos U \cos V=-\cos \beta(\cos L \cos \alpha-\sin L \sin \alpha) \\
& B_{\oplus}=-\cos U \sin V=-\cos \beta(\sin L \cos \alpha+\cos L \sin \alpha) \\
& C_{\oplus}=-\sin U=-\sin \beta
\end{aligned}
$$

Since the quantities $\alpha$ and $\beta$ are small, they may be expanded into series developments like $\sin x \approx x-x^{3} / 6$; the exact level of truncation must be determined. Now the series which gives $\cos \psi$ may be built, and we end up with about 32044 terms (see Table 4). That series must further be put as argument into $P_{20}(x)=\left(3 x^{2}-1\right) / 2$, which generates even more terms.

\section{Perturbation Method using several parameters}

We use here the Lie Transform (Deprit 1969) as canonical perturbation method, with the parameter $\epsilon$. The initial Hamiltonian (input) is written $\mathcal{H}^{(0)}=\sum_{i \geqslant 0} \frac{\epsilon^{i}}{i !} \mathcal{H}_{i}^{(0)}$; while the transformed Hamiltonian (output) is written $\mathcal{H}_{0}=\sum_{i \geqslant 0} \frac{\epsilon^{i}}{i !} \mathcal{H}_{0}^{(i)}$. This transformation is symbolized by the Lie triangle, which may be adapted to the case (2.1):

$$
\begin{aligned}
& \mathcal{H}_{0}^{(0)} \quad \mathcal{H}_{0}^{(0)}=-\frac{\mu^{2}}{2 L^{2}} \\
& \mathcal{H}_{1}^{(0)} \mathcal{H}_{0}^{(1)} \quad \mathcal{H}_{1}^{(0)}=-n_{\S} H \quad \mathcal{H}_{0}^{(1)} \\
& \mathcal{H}_{2}^{(0)} \quad \mathcal{H}_{1}^{(1)} \quad \mathcal{H}_{0}^{(2)} \quad \mathcal{H}_{2}^{(0)}+\delta \mathcal{H} \mathcal{B}_{2}^{(0)}+\gamma \mathcal{H} \mathcal{E}_{2}^{(0)} \quad \mathcal{H}_{1}^{(1)} \quad \mathcal{H}_{0}^{(2)}
\end{aligned}
$$

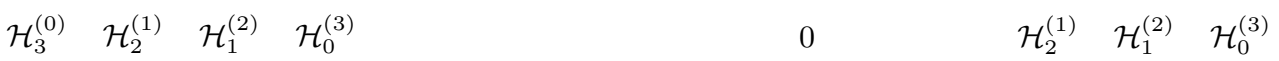

Figure 2. The classical Lie triangle and our specific Lie triangle.

The triangle is filled by the way of the following recursive formula, where the $\mathcal{W}_{k}$ are the generating functions and $(A ; B)$ is the Poisson parenthesis:

$$
\begin{aligned}
\mathcal{H}_{i}^{(j)} & =\mathcal{H}_{i+1}^{(j-1)}+\sum_{k=0}^{i} C_{i}^{k}\left(\mathcal{H}_{i-k}^{(j-1)} ; \mathcal{W}_{k+1}\right) \\
(A ; B) & =\sum_{i}\left(\frac{\partial A}{\partial q_{i}} \frac{\partial B}{\partial p_{i}}-\frac{\partial B}{\partial q_{i}} \frac{\partial A}{\partial p_{i}}\right)
\end{aligned}
$$


We may write $\mathcal{H}_{0}^{(i)}$ as $\overline{\mathcal{H}}_{0}^{(i)}$ in order to remember that the fast angle $l$ has been eliminated; we always put the periodic part in the generator $\mathcal{W}_{i}$.

For the first order: as $\mathcal{H}_{1}^{(0)}$ is already independent of $l$ here, we have $\mathcal{H}_{0}^{(1)}=\mathcal{H}_{1}^{(0)}=$ $-n_{\S} H$ and $\mathcal{W}_{1}=0$. For the second order, we have:

$$
\epsilon \mathcal{H}_{0}^{(2)}+\delta \mathcal{H} \mathcal{B}_{0}^{(2)}+\gamma \mathcal{H} \mathcal{E}_{0}^{(2)}=\epsilon \mathcal{H}_{2}^{(0)}+\delta \mathcal{H B}_{2}^{(0)}+\gamma \mathcal{H} \mathcal{E}_{2}^{(0)}+\left(\mathcal{H}_{0}^{(0)} ; \mathcal{W}_{2}\right)
$$

and we choose:

$$
\epsilon \overline{\mathcal{H}}_{0}^{(2)}+\delta \overline{\mathcal{H}}_{0}^{(2)}+\gamma \overline{\mathcal{H E}}_{0}^{(2)}=\frac{1}{2 \pi} \int_{0}^{2 \pi}\left(\epsilon \mathcal{H}_{2}^{(0)}+\delta \mathcal{H B}_{2}^{(0)}+\gamma \mathcal{H} \mathcal{E}_{2}^{(0)}\right) d l=\ldots
$$

while $\left(\mathcal{H}_{0}^{(0)} ; \mathcal{W}_{2}\right)$ reduces to $n \frac{\partial \mathcal{W}_{2}}{\partial l}$ which has then to be integrated with respect to $l$.

Higher orders may be achieved by the same way, provided we are able to compute the integrals. Some tricks are given in the literature, such as Aksnes (1971). The terms containing the factor $(f-l)$ receive a special treatment, as $(f-l)$ is indeed well known to play an important role in the problem of the artificial satellite (see Metris 1991). The

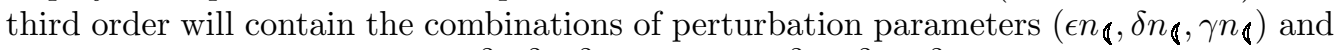
the fourth order will contain $\left(\epsilon^{2}, \delta^{2}, \gamma^{2}, \epsilon \delta, \epsilon \gamma, \delta \gamma, \epsilon n_{\S}^{2}, \delta n_{\S}^{2}, \gamma n_{\rrbracket}^{2}\right)$. We can easily select an isolated effect by putting the other parameters to zero.

\section{Results obtained by the symbolic manipulation software MM}

We used a specific FORTRAN code called the MM, standing for "Moon's series Manipulator", which has been developed at our University. In this tool, each expression is given by a series of linear trigonometric functions, with polynomial coefficients. The concern to keep linear expression is motivated by the fact that we want to keep easy integrations. An example of such a series is given in the Table 3 .

It is of course impossible to give all the results here, since the series may contain a lot of terms, but we give explicitly some of them here, and we comment the others. For example, we retrieve the known results for the first order effect of $J_{2}$ only. First, the expression of $\epsilon \overline{\mathcal{H}}_{0}^{(2)}$ is the same as the one given by $\left(-F_{1}^{*}\right)$ defined in (13) of Brouwer (1959), when setting $2 k_{2}=\epsilon$. Secondly, the expression of $\epsilon \mathcal{W}_{2}$ is also the same as the one given by $\left(-S_{1}\right)$ defined in (15) of Brouwer (1959). And thirdly, when writing the first order averaged equations of motion, we retrieve the two classical formulae giving the effect of $J_{2}$ on $g$ and $h$ (Szebehely 1989; Roy 1968; Jupp 1988). The associated peculiar value of the inclination which makes $\dot{\bar{g}}$ vanish, known as the critical inclination $I_{c}=63^{\circ} 26^{\prime}$, is quite famous (Szebehely 1989).

Some partial results (the terms in $\epsilon^{2}, \epsilon \delta$ and $\delta^{2}$ ) of the computation of the fourth-order averaged Hamiltonian are given explicitly in the Table 3 ; the series has been multiplied by a factor $\mathcal{F}=\frac{64 a^{2} \eta^{7}}{3 n^{2}}$ to make it more readable, and the variable $n_{\delta}$ represent the exponent in the perturbative parameter $\delta$ : for an expression of order $p+2$, we then have terms containing factors like $\epsilon^{p-n_{\delta}} \delta^{n_{\delta}}$. We retrieve also some known results for the effect of $J_{2}^{2}$ only: the expression of $\epsilon^{2} \overline{\mathcal{H}}_{0}^{(4)}$ is the same as the one given by $\left(-2 F_{2}^{*}\right)$ defined in (29) of Brouwer (1959). Moreover, the generator in $\epsilon^{2}$ has been computed and validated: the exact equivalence with Kozai's $S_{2}$ (given by equation (3.2) of Kozai 1962) has been established elsewhere, using the relationships of Shniad (1970) for the correspondence between generators of von Zeipel $\left(S_{i}\right)$ and the ones of Lie $\left(\mathcal{W}_{i}\right)$. 
Table 3. The series $\left(\epsilon^{2} \overline{\mathcal{H}}_{0}^{(4)}+\epsilon \delta \overline{\mathcal{H} \mathcal{Y}_{0}^{(4)}}+\delta^{2} \overline{\mathcal{H}}_{0}^{(4)}\right) \times \mathcal{F}$

\begin{tabular}{|c|c|c|c|c|c|c|c|c|c|c|c|c|c|}
\hline & $f$ & $g$ & $h$ & & $a$ & $n$ & $e$ & $\eta$ & $c$ & $s$ & $(f-l)$ & $n_{\delta} \mid$ & coefficient \\
\hline $\cos$ & 0 & 0 & 0 & 0 & 0 & 0 & 0 & 0 & 2 & 0 & 0 & 0 & $0.800 \times 10^{1}$ \\
\hline $\cos$ & 0 & 0 & 0 & 0 & 0 & 0 & 0 & 0 & 4 & 0 & 0 & 0 & $-0.400 \times 10^{2}$ \\
\hline $\cos$ & 0 & 0 & 0 & 0 & 0 & 0 & 0 & 1 & 0 & 0 & 0 & 0 & $-0.400 \times 10^{1}$ \\
\hline $\cos$ & 0 & 0 & 0 & 0 & 0 & 0 & 0 & 1 & 2 & 0 & 0 & 0 & $0.240 \times 10^{2}$ \\
\hline $\cos$ & 0 & 0 & 0 & 0 & 0 & 0 & 0 & 1 & 4 & 0 & 0 & 0 & $-0.360 \times 10^{2}$ \\
\hline $\cos$ & 0 & 0 & 0 & 0 & 0 & 0 & 2 & 0 & 0 & 0 & 0 & 0 & $0.500 \times 10^{1}$ \\
\hline $\cos$ & 0 & 0 & 0 & 0 & 0 & 0 & 2 & 0 & 2 & 0 & 0 & 0 & $-0.180 \times 10^{2}$ \\
\hline $\cos$ & 0 & 0 & 0 & 0 & 0 & 0 & 2 & 0 & 4 & 0 & 0 & 0 & $0.500 \times 10^{1}$ \\
\hline $\cos$ & 0 & 2 & 0 & 0 & 0 & 0 & 2 & 0 & 0 & 2 & 0 & 0 & $-0.200 \times 10^{1}$ \\
\hline $\cos$ & 0 & 2 & 0 & 0 & 0 & 0 & 2 & 0 & 2 & 2 & 0 & 0 & $0.300 \times 10^{2}$ \\
\hline $\cos$ & 0 & 0 & 2 & 0 & 0 & 0 & 0 & 0 & 0 & 2 & 0 & 1 & $-0.128 \times 10^{3}$ \\
\hline $\cos$ & 0 & 0 & 2 & 0 & 0 & 0 & 0 & 0 & 2 & 2 & 0 & 1 & $0.160 \times 10^{3}$ \\
\hline $\cos$ & 0 & 0 & 2 & 0 & 0 & 0 & 0 & 1 & 0 & 2 & 0 & 1 & $-0.480 \times 10^{2}$ \\
\hline $\cos$ & 0 & 0 & 2 & 0 & 0 & 0 & 0 & 1 & 2 & 2 & 0 & 1 & $0.144 \times 10^{3}$ \\
\hline $\cos$ & 0 & 0 & 2 & 0 & 0 & 0 & 2 & 0 & 0 & 2 & 0 & 1 & $-0.520 \times 10^{2}$ \\
\hline $\cos$ & 0 & 0 & 2 & 0 & 0 & 0 & 2 & 0 & 2 & 2 & 0 & 1 & $-0.200 \times 10^{2}$ \\
\hline $\cos$ & 0 & 2 & 2 & 0 & 0 & 0 & 2 & 0 & 0 & 0 & 0 & 1 & $-0.400 \times 10^{1}$ \\
\hline $\cos$ & 0 & 2 & 2 & 0 & 0 & 0 & 2 & 0 & 1 & 0 & 0 & 1 & $0.520 \times 10^{2}$ \\
\hline $\cos$ & 0 & 2 & 2 & 0 & 0 & 0 & 2 & 0 & 2 & 0 & 0 & 1 & $0.560 \times 10^{2}$ \\
\hline $\cos$ & 0 & 2 & 2 & 0 & 0 & 0 & 2 & 0 & 3 & 0 & 0 & 1 & $-0.600 \times 10^{2}$ \\
\hline $\cos$ & 0 & 2 & 2 & 0 & 0 & 0 & 2 & 0 & 4 & 0 & 0 & 1 & $-0.600 \times 10^{2}$ \\
\hline $\cos$ & 0 & 2 & -2 & 0 & 0 & 0 & 2 & 0 & 0 & 0 & 0 & 1 & $-0.400 \times 10^{1}$ \\
\hline $\cos$ & 0 & 2 & -2 & 0 & 0 & 0 & 2 & 0 & 1 & 0 & 0 & 1 & $-0.520 \times 10^{2}$ \\
\hline $\cos$ & 0 & 2 & -2 & 0 & 0 & 0 & 2 & 0 & 2 & 0 & 0 & 1 & $0.560 \times 10^{2}$ \\
\hline $\cos$ & 0 & 2 & -2 & 0 & 0 & 0 & 2 & 0 & 3 & 0 & 0 & 1 & $0.600 \times 10^{2}$ \\
\hline $\cos$ & 0 & 2 & -2 & 0 & 0 & 0 & 2 & 0 & 4 & 0 & 0 & 1 & $-0.600 \times 10^{2}$ \\
\hline $\cos$ & 0 & 0 & 0 & 0 & 0 & 0 & 0 & 0 & 0 & 0 & 0 & 2 & $-0.176 \times 10^{3}$ \\
\hline $\cos$ & 0 & 0 & 0 & 0 & 0 & 0 & 0 & 0 & 2 & 0 & 0 & 2 & $0.384 \times 10^{3}$ \\
\hline $\cos$ & 0 & 0 & 0 & 0 & 0 & 0 & 0 & 0 & 4 & 0 & 0 & 2 & $-0.800 \times 10^{2}$ \\
\hline $\cos$ & 0 & 0 & 0 & 0 & 0 & 0 & 0 & 1 & 0 & 4 & 0 & 2 & $-0.720 \times 10^{2}$ \\
\hline $\cos$ & 0 & 0 & 0 & 0 & 0 & 0 & 2 & 0 & 0 & 0 & 0 & 2 & $-0.540 \times 10^{2}$ \\
\hline $\cos$ & 0 & 0 & 0 & 0 & 0 & 0 & 2 & 0 & 2 & 0 & 0 & 2 & $0.156 \times 10^{3}$ \\
\hline $\cos$ & 0 & 0 & 0 & 0 & 0 & 0 & 2 & 0 & 4 & 0 & 0 & 2 & $0.100 \times 10^{2}$ \\
\hline $\cos$ & 0 & 0 & 4 & 0 & 0 & 0 & 0 & 0 & 0 & 4 & 0 & 2 & $-0.800 \times 10^{2}$ \\
\hline $\cos$ & 0 & 0 & 4 & 0 & 0 & 0 & 0 & 1 & 0 & 4 & 0 & 2 & $-0.720 \times 10^{2}$ \\
\hline $\cos$ & 0 & 0 & 4 & 0 & 0 & 0 & 2 & 0 & 0 & 4 & 0 & 2 & $0.100 \times 10^{2}$ \\
\hline $\cos$ & 0 & 2 & 0 & 0 & 0 & 0 & 2 & 0 & 0 & 2 & 0 & 2 & $-0.360 \times 10^{2}$ \\
\hline $\cos$ & 0 & 2 & 0 & 0 & 0 & 0 & 2 & 0 & 2 & 2 & 0 & 2 & $0.600 \times 10^{2}$ \\
\hline $\cos$ & 0 & 2 & 4 & 0 & 0 & 0 & 2 & 0 & 0 & 2 & 0 & 2 & $0.300 \times 10^{2}$ \\
\hline $\cos$ & 0 & 2 & 4 & 0 & 0 & 0 & 2 & 0 & 1 & 2 & 0 & 2 & $0.600 \times 10^{2}$ \\
\hline $\cos$ & 0 & 2 & 4 & 0 & 0 & 0 & 2 & 0 & 2 & 2 & 0 & 2 & $0.300 \times 10^{2}$ \\
\hline $\cos$ & 0 & 2 & -4 & 0 & 0 & 0 & 2 & 0 & 0 & 2 & 0 & 2 & $0.300 \times 10^{2}$ \\
\hline $\cos$ & 0 & 2 & -4 & 0 & 0 & 0 & 2 & 0 & 1 & 2 & 0 & 2 & $-0.600 \times 10^{2}$ \\
\hline $\cos$ & 0 & 2 & -4 & 0 & 0 & 0 & 2 & 0 & 2 & 2 & 0 & 2 & $0.300 \times 10^{2}$ \\
\hline
\end{tabular}

All these checks and some symmetry considerations (especially by the way of the tracer $\eta$ ) give good confidence in the new results, which contain other effects than $J_{2}$; for example, the cross effect $\epsilon \delta$ may be rewritten in full as: 


$$
\begin{array}{rrrll}
\overline{\mathcal{H Y}}_{0}^{(4)} \times \mathcal{F}=\left[\begin{array}{rcrl}
32 & & s^{2}\left(5 c^{2}-4\right) & \cos (2 h) \\
+48 & \eta & s^{2}\left(3 c^{2}-1\right) & \cos (2 h) \\
-4 & e^{2} & s^{2}\left(5 c^{2}+13\right) & \cos (2 h) \\
-4 & e^{2} & & (\cos (2 g+2 h)+\cos (2 g-2 h)) \\
+52 & e^{2} & c & (\cos (2 g+2 h)-\cos (2 g-2 h)) \\
+56 & e^{2} & c^{2} & (\cos (2 g+2 h)+\cos (2 g-2 h)) \\
-60 & e^{2} & c^{3} & (\cos (2 g+2 h)-\cos (2 g-2 h)) \\
-60 & e^{2} & c^{4} & (\cos (2 g+2 h)+\cos (2 g-2 h))
\end{array}\right]
\end{array}
$$

A critical issue by making such computations is the rapid growth of the number of terms in the series (typically ten thousands of terms here). Needless to say, quite a big work of simplification has to be made, especially because of the redundancy $(\eta \leftrightarrow e)$ and $(c \leftrightarrow s)$ of the set (2.6). Among these simplifications are some series of the kind $\left(e^{2} \eta^{-7}-e^{2} \eta^{-9}+e^{4} \eta^{-9}\right)$ which in fact cancel to zero. Some substitutions like $\left(s^{2} \rightarrow 1-\right.$

\begin{tabular}{|c|c|c|c|c|c|c|c|c|c|c|c|c|c|}
\hline & $f$ & $g$ & $h$ & $L$ & $D$ & $l^{\prime}$ & $l$ & $F$ & $V e$ & $T e$ & $c$ & $s$ & coefficient \\
\hline $\min$ & 1 & 1 & -1 & -1 & -12 & -4 & -9 & -8 & -54 & -48 & 0 & 0 & $0.20037083 \times 10^{-14}$ \\
\hline $\max$ & 1 & 1 & 1 & 1 & 12 & 4 & 9 & 8 & 54 & 48 & 1 & 1 & $0.49740618 \times 10^{0}$ \\
\hline
\end{tabular}
$c^{2}$ ) for example may cancel a lot of terms, while the reverse operation of combining some terms may also make the expressions more compact, all simplifications being made before. The third body perturbation series contains quite a lot of terms; the series expressing $\cos (\psi)$ only is already about 32044 terms. We show in the Table 4 the extreme coefficients of the trigonometric variables and the extreme exponents of the polynomial variables which appear during the computation of $\cos (\psi)$.

Table 4. Extreme coefficients (or exponents) appearing in the computation of $\cos (\psi)$.

Numerical accuracy considerations are also required (we are working in double precision) and some terms must therefore be removed. We may also always reject during the computation the terms which are of lower magnitude than others, by a skilful choice.

\section{Conclusions}

We have achieved, among others, second order results for the combined effect of $J_{2}$ and $C_{22}$, and introduced the third body effect. The averaged Hamiltonians and the generators for the first and second order effects of $J_{2}$ have been validated by comparison with the results of Brouwer (1959) and Kozai (1962). More detailed results will be published in a forthcoming paper.

We could add even more perturbations (Sun, other lunar harmonics like $C_{31}$, librations, etc.) provided our symbolic manipulation software is able to handle them. We could also eliminate the next fastest angle $(g$ or $h)$ in order to obtain the secular motion. One future aim is to validate theoretically these results by numerical integrations of the several 
effects. An experimental validation could also be followed by a comparison with data of a real lunar satellite.

\section{References}

Aksnes, K. 1971, Celest. Mech. \&J Dyn. Astr. 4, 119-121.

Bills, B., Ferrari, A. 1980, J. Geophys. Res. 85, 1013-1025.

Brouwer, D. 1959, Astron. J. 64, 378-397.

Chapront-Touzé, M., Chapront, J. 1991, Lunar Tables and Programs 4000 BC to AD 8000. Willmann-Bell.

Cook, A. 1988, The Motion of the Moon. IOP Publishing Ltd.

Deprit, A. 1969, Celest. Mech. \& Dyn. Astr. 1, 12-30.

Jupp, A. 1988, Celest. Mech. \& Dyn. Astr. 43, 127-138.

Kaula, W. 1966, Theory of Satellite Geodesy. Blaisdell Publishing Company.

Konopliv, A., Asmar, S., Carranza, E., Sjogren, W., Yuan, D. 2001, Icarus 150, 1-18.

Kozai, Y. 1962, Astron. J. 67, 446-461.

Metris, G. 1991, Celest. Mech. \& Dyn. Astr. 52, 79-84.

Roy, A. 1968, Icarus 9, 82-132.

Shniad, H. 1970, Celest. Mech. \& Dyn. Astr. 2, 114-120.

Szebehely, V. 1989, Adventures in Celestial Mechanics. University of Texas Press.

\section{Discussion}

Mikhail Marov: I am just wondering: You limited yourself only to the J2 harmonic. What about other even harmonics, say 4, say 6 , and some others. The lunar field is quite well known at a very high order of expansion. Why did you neglect it?

Bernard De SAedeleer: We can't always take it into account. In fact, I just submitted an article to Celestial Mechanics which computes the average Hamiltonian for the horizontal effect, even or odd. You can always introduce more and more terms, if you want. You just have to compute the corresponding Hamiltonian. It can be done.

Mikhail Marov: But at the beginning you mentioned you also took into account the mascons. I guess at a level of J2 is hardly possible to account for the mascons.

Bernard De Saedeleer: No. Of course, the J2 is just a one big feature of the potential. If you want really to have the resolution of the mascons ...

Mikhail Marov: ... more subtle effects?

Bernard De Saedeleer: Yes. If you want to have more precise effects, you go into more numerical analysis - it becomes more numerical. If you want to have a model $16 \times 16$, you have all those coefficients, and it becomes more and more complicated.

Mikhail MARov: If you will limit yourself with such a theory, it will be hardly possible to predict accurately orbits - especially the low orbit lunar satellites.

Bernard De SAEDeleer: Yes, you can always refine it. 